\title{
TES Bolometer Array for the APEX-SZ Camera
}

\author{
J. Mehl • P.A.R. Ade · K. Basu • D. Becker • A. Bender · F. Bertoldi • H.M. Cho • \\ M. Dobbs • N.W. Halverson · W.L. Holzapfel • R. Gusten • J. Kennedy • \\ R. Kneissl - E. Kreysa - T.M. Lanting • A.T. Lee • M. Lueker • K.M. Menten • \\ D. Muders • M. Nord · F. Pacaud • T. Plagge · P.L. Richards · P. Schilke • \\ D. Schwan · H. Spieler $\cdot$ A. Weiss $\cdot$ M. White
}

Received: 23 July 2007 / Accepted: 15 September 2007 / Published online: 25 January 2008 (C) Springer Science+Business Media, LLC 2008

\begin{abstract}
We will report on the APEX-SZ bolometer camera which houses a 320 element Transition-Edge Sensor (TES) bolometer array designed to survey for galaxy clusters using the 12-meter diameter APEX telescope sited in Chile. Design and fabrication of the TES bolometer array will be discussed, as well as its integration with a frequency-domain SQUID multiplexed readout system. The full configuration of the APEX-SZ camera was deployed in April 2007. A preliminary galaxy cluster map from this deployment will be presented.
\end{abstract}

\footnotetext{
J. Mehl (凶) · W.L. Holzapfel · A.T. Lee · M. Lueker · T. Plagge · P.L. Richards · D. Schwan · M. White 
Keywords Bolometer · Transition edge sensor · SQUID · Multiplexer · Sunyaev-Zel'dovich · CMB

PACS 07.57.Kp $\cdot$ 85.25.Dq $\cdot 85.25 . \mathrm{Oj} \cdot 85.25 . \mathrm{Pb} \cdot 98.80 . \mathrm{Es} \cdot 95.85 . \mathrm{Bh} \cdot 98.65 . \mathrm{Cw}$

\section{Introduction}

Studies of galaxy clusters can provide a wealth of cosmological data about the largescale structure of the universe and the nature of dark energy. The hot electron gas within a galaxy cluster scatters cosmic microwave background (CMB) photons in a process known as the Sunyaev-Zel'dovich (SZ) effect [1]. Unlike X-ray or optical measurements, this effect is largely independent of cluster distance or redshift, and provides a powerful means to detect new galaxy clusters. Because the sensitivity of individual bolometers has reached fundamental limits, large bolometer arrays are necessary to efficiently map the SZ effect on large regions of the sky.

Spiderweb absorber TES bolometers are well-suited to fabrication in large arrays and are capable of being instrumented with a multiplexed SQUID readout system. We have designed and fabricated a 320 element TES bolometer array which is cooled to $250 \mathrm{mK}$ using the combination of a three-stage He sorption fridge and mechanical pulse tube cooler. The detector array is instrumented with a frequency domain SQUID multiplexing readout system. The camera is mounted on the $12 \mathrm{~m}$ APEX Telescope [2], located at $5100 \mathrm{~m}$ altitude on the Atacama plateau in Chile.

\section{Array Design and Fabrication}

The APEX-SZ array consists of six wedges, each with $55150 \mathrm{GHz}$ pixels (see Fig. 1). The array was fabricated in the Berkeley Microlab cleanroom facility [3]. Each bolometer has a $3 \mathrm{~mm}$ diameter gold spiderweb absorber. The spiderweb geometry is chosen to reduce the heat capacity of the absorber to minimize the optical time constant, and to reduce the cross-section of the bolometer to cosmic ray hits. The gold web has a DC sheet resistance of $\sim 200 \Omega / \square$, which is not the ideal resistance for maximum absorption in a cavity, but is attained from a film thickness that is feasible to deposit in a thermal evaporator. An Al/Ti bilayer TES is located at the center of each web; the thermal spreading time in the web sets the optical time constant $\tau_{\mathrm{opt}} \sim 9 \mathrm{~ms}$. The bilayer metal thicknesses are chosen to give $T_{\mathrm{c}} \sim 450 \mathrm{mK}$ and $R_{\mathrm{n}} \sim 1.2 \Omega$. The same aluminum layer that is in the TES is also used for bias leads. A gold finger runs parallel to the bias leads, and is used to tune the thermal conductivity of the bolometer to $G \sim 200 \mathrm{pW} / \mathrm{K}$. The bolometer is suspended on a $1 \mu \mathrm{m}$ thick low-stress $\mathrm{Si}_{3} \mathrm{~N}_{4}$ membrane. The bolometer is thermally isolated from the bath by etching the $\mathrm{Si}$ underneath the silicon nitride web with a dry $\mathrm{XeF}_{2}$ gas etch.

The bolometers are horn-coupled, with the lower band edge set by a waveguide cutoff. The upper band edge is set by metal-mesh filters. To maximize physical yield and ease of fabrication, we do not remove most of the silicon behind the bolometers. Instead, for currently deployed APEX-SZ detectors, wafer thickness is chosen 
to create a $3 \lambda / 4$ backshort in the silicon wafer, which is metalized on the backside and mounted to an invar plate. Extensive finite-element analysis simulations in HFSS [4] have shown that we can achieve good optical coupling with low optical crosstalk using this geometry. Tests in the APEX-SZ receiver have confirmed that pixel-to-pixel crosstalk is on the order of $1-2 \%$, which is consistent with the simulations. Fourier transform spectroscopy (FTS) measurements in the APEX-SZ receiver show that our pixels have a $23 \mathrm{GHz}$ bandwidth properly centered at $150 \mathrm{GHz}$. This is narrower for than our designed bandwidth of $38 \mathrm{GHz}$. FTS measurements in other receivers, however, suggest that bolometers with the same geometry as the APEX-SZ pixels have the correct bandwidths that match the simulations. Preliminary simulations suggest that a narrow bandwidth may be due to a high RF sheet resistance for our spiderweb absorbers with a $3 \lambda / 4$ backshort. We are investigating whether something in our fabrication process has caused a large increase in the absorber resistance. We also intend to further test the other elements in the optical path of the APEX-SZ receiver for non-bolometer mechanisms causing a narrow bandwidth.

For future generations of spiderweb bolometers, we intend to use thinner $\mathrm{Si}$ wafers that will give a $\lambda / 4$ backshort, which should improve the bandwidth performance of the detectors. We also intend to make some refinements to the horn-array to improve the waveguide to bolometer cavity coupling. HFSS simulations have shown that having a slight conical flare at the bolometer side of the waveguide improves the overall bandwidth performance, especially at low frequencies near the waveguide cutoff.

\section{Readout and System Integration}

The APEX-SZ bolometer array is instrumented by a frequency domain multiplexer readout system (fMUX), designed and built in Berkeley [5]. A comb of AC carriers, with frequencies ranging from $200 \mathrm{kHz}$ to $1 \mathrm{MHz}$, bias bolometers in series with an LC resonant circuit that selects the bias for the bolometer. Bolometer signals appear as amplitude modulated sidelobes to the carriers. The summed current, with the carriers suppressed by a nulling signal $180^{\circ}$ out-of-phase with the carrier comb, is measured with a 100-element SQUID array, operated with shunt feedback. An ana$\log$ demodulator recovers the observation signal from each sensor.

Early APEX-SZ bolometers were unstable when instrumented with the fMUX system. The bolometers would latch superconducting as soon as they entered the superconducting transition. This problem occurred because the bias circuit of the bolometer contains inductance which slows the delivery of bias power to the TES. A conservative stability criterion [6] states that the following relation must be true for the TES to remain stable:

$$
\frac{\tau_{\text {sensor }}}{\tau_{\text {bias }}}>5.8
$$

where $\tau_{\text {sensor }}=\tau_{0} /(\mathcal{L}+1)$ is the effective bolometer time constant, $\mathcal{L}$ is the TES loop gain, and $\tau_{\text {bias }}=2 L_{\mathrm{MUX}} / R \sim 50 \mu \mathrm{s}$ is the AC bias circuit time constant for the current fMUX system. The early APEX-SZ bolometers had an intrinsic time constant $\tau_{0}=C / G \sim 100 \mu \mathrm{s}$, so that they became unstable as soon as they entered the superconducting transition. This is consistent with actual bolometer performance. We 


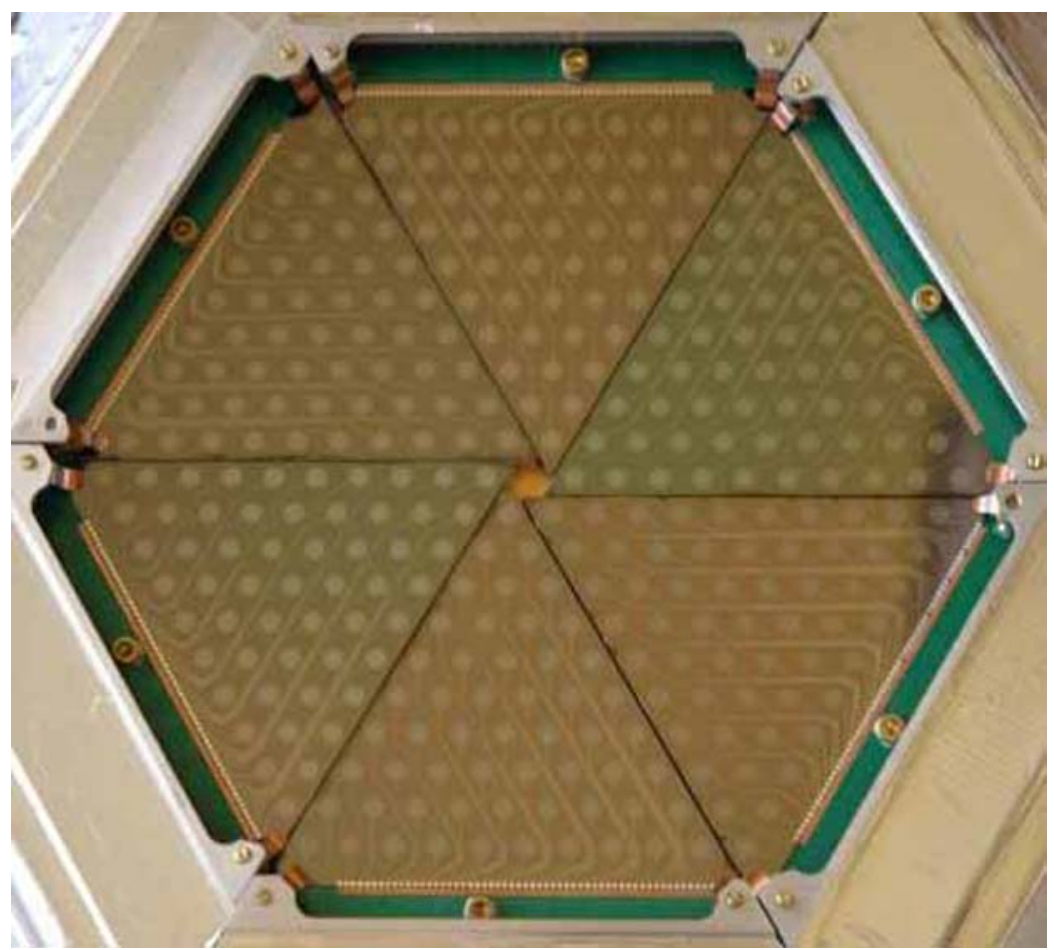

Fig. 1 (Color online) The full 320 element APEX-SZ array, deployed in April 2007

added a $3 \mu \mathrm{m}$ thick gold ring to the bolometers to increase the heat capacity, which increased the intrinsic time constant to $\tau_{0} \sim 30 \mathrm{~ms}$. This allows stable bolometer operation deep into the superconducting transition. The added gold heat capacity must remain in strong thermal contact with the TES for stability to be maintained.

With the TES bolometers stabilized, we achieve the expected noise performance of roughly $100 \mathrm{aW} / \mathrm{Hz}^{1 / 2}$. The noise is white for frequencies of interest for galaxy cluster surveys. The $1 / \mathrm{f}$ knee of our noise is typically at $1-2 \mathrm{~Hz}$.

\section{Results}

In April 2007 the full APEX-SZ array was deployed at the APEX telescope in Chile for two weeks of observation time. The APEX-SZ team was able to keep a $75 \%$ duty cycle on the sky during this observation period. The other $25 \%$ of time was lost to helium sorption fridge cycling and receiver tuning. Observations included planet scans on Mars, Jupiter, and Saturn, quasars, galactic HII regions, known galaxy clusters, and blank-sky fields. Future scientific goals for the APEX-SZ receiver include observing high-redshift galaxy clusters, SZ mapping nearby extended clusters, and measuring the SZ power spectrum at arcminute scales.

Sophisticated analysis techniques are required to map the very dim SZ galaxy cluster signal. For instance, even though the APEX site is one of the best known sites 




Fig. 2 Temperature map of the galaxy cluster Abell 2163

for (sub)mm astronomy and the weather during our observations was good, the dominant signal in our detectors is atmospheric fluctuations. We use principal component analysis (PCA) to quantify and remove the correlated atmospheric noise signal from the bolometer timestreams. Residual atmospheric signals, after applying our PCA removal algorithm, are well below the dark noise of each sensor.

Our data analysis pipeline is sufficiently mature that we are able to make well understood and calibrated maps of known galaxy clusters. Figure 2 shows a map of the cluster Abell 2163. This map was made with roughly one hour of telescope integration time. The next step for the analysis project will be to produce maps of our blank-sky fields and further refine the $1 / \mathrm{f}$ noise removal in all of our maps.

The technology used in APEX-SZ has also been deployed at the South Pole Telescope (SPT), on a much larger scale. The SPT bolometer array has 1000 pixels with three observation bands centered at 95, 150, and $220 \mathrm{GHz}$. The South Pole telescope is fully dedicated to SZ camera observations for the entire South Pole winter observing season, allowing for a large amount of observation time. Refinements are currently being made to the bolometer array design and fabrication for future seasons of APEX-SZ and SPT observations.

Acknowledgements We would like to thank E. Shirokoff and X.F. Meng for bolometer fabrication assistance. This work is supported by the National Science Foundataion under Grant No. AST-0138348. Work at L.B.N.L. is supported the Director, Office of Science, Office of High Energy and Nuclear Physics, of the U.S. Department of Energy under Contract No. DE-AC02-05CH11231. 


\section{References}

1. R.A. Sunyaev, Ya.B. Zeldovich, Astrophys. Space Sci. 7, 3 (1970)

2. R. Gusten, L.A. Nyman, P. Schilke, K. Menten, C. Cesarsky, R. Booth, Astron. Astrophys. 454, L13 (2006)

3. Berkeley Microlab, URL http://www.microlab.berkeley.edu

4. Ansoft Corporation, URL http://www.ansoft.com/products/hf/hfss/

5. T.M. Lanting, H.-M. Cho, J. Clarke, M.A. Dobbs, A.T. Lee, M. Lueker, P.L. Richards, A.D. Smith, H.G. Spieler, Nucl. Methods Phys. Res. A 520, 548 (2004)

6. K.D. Irwin, G.C. Hilton, D.A. Wollman, J.M. Martinis, J. Appl. Phys. 83, 3978 (1998) 